\title{
Dhamira za Maandishi ya Kuta za Vyooni Jijini Dar es Salaam
}

\author{
Willy Migodela ${ }^{1}$
}

\section{Ikisiri}

Makala haya yanahusu dhamira za maandishi, hususani ya vyooni Jijini Dar es Salaam. Lengo ni kuangalia dhamira za maandishi hayo na nafasi yake kwa jamii. Katika uchambuzi tumetathmini ubunifu unaojitokeza katika maandishi hayo. Aidha, kwa kutumia mbinu za utafiti maktabani kama vile uchambuzi matini na mbinu za uwandani, tulipata data muafaka kuhusu suala la kiutafiti lililoshughulikiwa katika makala haya. Pia, tumedurusu maandiko mbalimbali yanayozungumzia dhamira katika maandishi yaliyoandikwa sehemu mbalimbali na namna lugha ilivyotumika kutoa mawasiliano. Aidha, tumetumia nadharia ya Elimumitindo ambayo ndiyo inayoongoza uchambuzi na mjadala katika makala haya. Hali kadhalika, kutokana na utafiti huu, imebainika kuwa dhamira zilizojitokeza zaidi ni zile zinazohusu maradhi, maarifa, maadili na dini. Ilibainika pia kuwa dhamira hizi zinaakisi muundo na mfumo wa maisha ya jamii husika. Kutokana na matokeo haya, imehitimishwa kuwa ubunifu uliopo kwenye maandiko ya vyooni unasawiri hali mbalimbali katika jamii kama vile mtazamo wa wanajamii kuhusu maana na umuhimu wa elimu, dini, maadili na utamaduni kwa ujumla. Hatimaye, katika makala haya tutaona jinsi lugha safihi (matumizi ya lugha yasiyo na adabu) inavyojitokeza katika maandishi ya vyooni.

Istilahi Muhimu: dhamira; ubunifu; elimu-mitindo; maandiko ya vyooni; lugha safihi DOI: https://doi.org/10.37759/mjed.2017.1.1.2

\section{Utangulizi}

Makala haya yanahusu dhamira za maandishi katika kuta za vyoo. Utafiti huu ulifanyika katika vyoo vya wanaume mkoani Dar es Salaam, ukiwa umehusisha vyoo vya baadhi ya Vyuo Vikuu Dar es Salaam, baadhi ya shule za sekondari, shule za msingi na vyoo vya umma kama vile vya stendi na sokoni, kwani kwa kiasi kikubwa katika maeneo hayo kuna maandishi mengi ambayo huandikwa na watumiaji wa vyoo hivyo.

Mtafiti ameshughulikia maandishi yaandikwayo katika kuta za vyoo, na hasa maandishi ambayo huandikwa katika kuta ndani ya vyoo husika. Maandishi hayo ambayo mengine huambatana na michoro yana lugha iliyosheheni usanaa hata kuweza kuvuta hamu ya mtafiti wa lugha za kisanaa kufanya utafiti huu. Lugha ya kisanaa ndiyo hubeba tofauti ya msingi kati ya maandishi yanayoitwa ya kifasihi na yale ambayo si ya kifasihi. Kutokana na ukweli huu, mtafiti alichunguza ili kubaini ikiwa kuna ufasihi katika maandishi hayo, na kujaribu kuunda kiunzi cha kinadharia kuelezea uandishi huo.

Katika ujumla wake, maandishi na michoro iliyopo katika sehemu za umma, hasa kutani na kwenye kingo za madaraja, huitwa graffiti. Graffiti asili yake ni neno la Kirumi graffiato,

\footnotetext{
${ }^{1}$ Chuo Kikuu Kishiriki cha Askofu Mkuu Yakobo (SAUT-Songea); Email: $\underline{\text { migodela@ hotmail.com }}$
} 
linalomaanisha michoro na maandishi mbalimbali yaliyochorwa katika sehemu mbalimbali za umma. Nchini Ugiriki dhana hii iliitwa graphein, likiwa na maana ya kuandika. Warumi wanaamini graphein ndiyo asili ya graffiti. Katika lugha ya Kiswahili neno hili tumelitohoa na kuliita grafiti. Tunaweza kuilinganisha etimolojia ya neno hili grafiti na lile neno la Kiingereza "literature" ambalo chimbuko lake ni huko huko Ulaya. "Literature" ambayo ilifasiliwa kuwa ni "Fasihi" katika Kiswahili imetoholewa kutoka neno la Kilatini littera ambalo maana yake ni "herufi" au "uandikaji”. Katika Kiswahili, neno Fasihi linabeba dhana pana zaidi ambayo inaunganisha kwa pamoja maandishi na ufasaha wa lugha ambao si lazima uwemo katika maandishi. Hivyo, ikiwa katika grafiti tunapata maandishi na ufasaha wa maandishi hayo, kwa hakika tunaweza kuitafiti fasihi ndani yake. Suala hili ndilo limekuwa chimbuko la utafiti huu, huku tukijiuliza swali, je maandishi katika vyoo yanaweza kuwa ni ya kifasihi? Au kwa lugha nyingine, je, hizi grafiti katika vyoo zinaweza kubeba ujumbe uliowekwa kisanaa na hata kuhalalisha kuitwa fasihi?

Mutembei (2005) alichunguza matumizi ya lugha katika mabango yenye ujumbe wa UKIMWI barani Afrika. Alitumia nadharia ya Semiotiki ya kiutamaduni ili kuona jinsi jamii itumiavyo lugha. Mtafiti aligundua ufasihi upatikanao katika mabango hayo. Vipengele vilivyodhihirisha ufasihi ni matumizi ya sitiari, usambamba na nudhumu. Fischer (2009) alifanya utafiti wake kuhusu mawasiliano yanavyoweza kufanyika kwa kutumia maandishi ya vyooni. Utafiti wake aliufanya katika vyoo vya wanawake. Fischer aligundua mijadala mbalimbali inayohusu masuala ya kidini, mapenzi na uhusiano (urafiki). Katika mawasiliano hayo lugha inayotumika ni lugha ya kishairi, lugha ya nathari, maswali, majibu na mara chache kuna mafumbo. Alihitimisha kwa kupata grafiti zenye maandishi yenye adabu, na yenye kutoa mwelekeo wa uhuru wa maamuzi kwa wasiojiamini. Alifanya utafiti wake kwa kuegemea kipengele cha mawasiliano na uharabu. Utafiti huo haukuwa wa kifasihi, lakini unatoa mwanga na mwelekeo mzuri katika makala haya.

\section{Mbinu za Utafiti}

Mbinu zilizotumika katika utafiti huu ni pamoja na usanisi wa utafiti, populesheni ya utafiti, sampuli na usampulishaji, na mbinu za ukusanyaji wa data.

\subsection{Usanisi wa Utafiti}

Kothari (2008) anasema kwamba usanisi ni mpango au mpangilio unaoonesha jinsi na namna data zitakavyokusanywa na kuchambuliwa kwa kutumia gharama nafuu zaidi. Sababu kuu ya kuchagua mbinu hii ya uchunguzi kifani ni kuwa inatoa fursa ya kuchunguza jambo kwa undani zaidi na kuhusisha vipengele mbalimbali vinavyounda ama kujenga jambo hilo (Isaac \& Michael, 1981; Creswell, 2009). Hii ndiyo sehemu inayounganisha kazi nzima ya utafiti kwani ndicho kiungo cha kazi nzima.

\subsection{Populesheni na Sampuli ya Utafiti}


Populesheni ni idadi ya watu, makundi ya watu au vitu vyote ambavyo mtafiti hutumia kukamilisha utafiti wake (Babbie, 1999). Populesheni lengwa ya utafiti huu ni kuta za vyoo kumi ambapo vinne ni kutoka Manispaa ya Ilala na sita kutoka Manispaa ya Kinondoni. Vyoo hivi vinajumuisha vile vilivyopo katika sehemu za umma kama vile sokoni, kituo cha basi, shuleni na vyuoni. Sababu ya kuwa na mchanganyiko huu wa vyoo ni kuwezesha kupatikana data zenye mitazamo mbalimbali ya watumiaji wake.

Makala haya yametumia data za msingi. Data za msingi ni zile zinazokusanywa kwa mara ya kwanza na hazijawahi kukusanywa na mtafiti mwingine kwa minajili ya uchambuzi. Hivyo, data hizi ni halisi (Kothari, 1992). Kwa msingi huu basi, data za msingi za makala haya zilikusanywa kutoka katika kuta za vyoo kumi vilivyopo Manispaa za Ilala na Kinondoni. Lengo la kuchagua vyoo hivyo ni kwamba vitasaidia kutoa data zitakazokidhi mahitaji ya makala haya.

\subsection{Mbinu za Ukusanyaji wa Data}

Mbinu iliyotumika katika utafiti huu ni uchambuzi matini. Uchambuzi matini ni mbinu ya kukusanya data ambapo mtafiti husoma matini au maandishi ya kitaaluma na yasiyo ya kitaaluma na kisha kubaini data na taarifa zihusuzo utafiti wake na kuzirekodi katika shajara (Kothari, 2008). Hivyo basi, mbinu hii imetumika kukusanya data za msingi. Mtafiti aliingia katika vyoo lengwa na kupiga picha maandishi yaliyoko katika kuta za vyoo hivyo kwa kamera. Baada ya hapo, aliyahamishia katika sharaja maalumuu iliyoandaliwa na kisha kusoma data moja baada ya nyingine. Katika usomaji wa data hizo ndipo data za kukidhi mahitaji ya makala haya zilipatikana. Kwa mantiki hiyo, mbinu hizi zimetuwezesha kubaini maandishi yaliyoandikwa katika kuta za vyoo yenye dhamira mbalimbali na kuonesha nafasi ya dhamira hizo kwa jamii..

\section{Kiunzi cha Nadharia}

Makala haya yameongozwa na nadharia ya Elimu-mitindo. Uteuzi wa nadharia hii ulifanyika kutokana na utofauti wa mitindo ya uandishi unaopatikana kutoka kwa waandishi wa maandishi ya vyoo. Enkvist (1973) anasema kuwa elimu mitindo ni kaida za utofauti kati ya kazi mbalimbali za kisanaa kama ilivyo kwenye ushairi, riwaya na tamthiliya. Anaendelea kusema kuwa hatuwezi kujua mtindo wa mwandishi kwa kuangalia kazi yake moja tu, bali kwa kutumia kazi zake mbalimbali ndipo tutangundua mtindo wa mwandishi husika. Kwa jumla, tunaweza kusema kuwa mtindo ni namna lugha inavyoweza kutumika tofauti tofauti kuendana na muktadha na lengo. Hivyo, kwa kuangalia mtindo wa maandishi ya vyoo itasaidia kubainisha dhamira zinazoibuka kutoka kwa waandishi wa vyooni na nafasi za dhamira hizo katika jamii.

Miall na Kuiken (1994) wanasema kuwa elimu mitindo ni miongoni mwa vipengele muhimu sana vinavyotofautisha baina ya kazi za kifasihi na zile zisizo za kifasihi. Kazi kama vile za ushairi hutofautiana na maandishi mengine kama ya hotuba na risala. Mitindo inayotumika 
kwenye kazi ya Fasihi ni tofauti na ile ya mawasiliano ya kawaida. Hivyo, nadharia hii imetuongoza katika uchambuzi wetu wa data, kwa minajili ya kudadavua mtindo wa maandishi ya vyooni ili kubainisha dhamira zinazoibuka kutokana na mitindo hiyo na nafasi za dhamira hizo katika jamii.

\section{Mapitio ya Maandiko kuhusu Maandishi ya Sehemu za Umma na Chooni}

Watafiti mbalimbali wamefanya tafiti juu ya dhamira za maandishi ya sehemu za umma Tanzania. Mahenge (2009) alifanya uchunguzi wa fani na maudhui katika semi zilizoandikwa katika tiketi za daladala Dar es salaam. Daladala ni usafiri wa umma. Hivyo, maandishi yanayopatikana kwenye tiketi ni moja ya maandishi ya sehemu za umma. Katika fani, alichunguza baadhi ya vipengele kuhusiana na misemo iliyoko katika tiketi hizo. Vipengele hivyo ni tamathali za usemi, sitiari, metonimia, tashbiha, tashihisi, picha, takriri na wahusika. Vipengele vya maudhui alivyovichunguza ni dhamira. Alipata dhamira kuhusu maradhi ya UKIMWI, imani kwa Mungu, ibada, kazi na sala. Pia, miongozo ya maisha, wajibu wa abiria, ushoga kuwa laana, umuhimu wa tiketi, umuhimu wa kufanya kazi, tabia njema, subira, ukarimu, maadili, ukweli wa maisha na mapenzi. Vipengele vingine vya maudhui ni pamoja na falsafa, itikadi, migogoro na ujumbe.

Baada ya kuchambua fani na maudhui, kimaudhui, mtafiti alithibitisha kuwa misemo iliyopo kwenye tiketi za daladala inaakisi maisha ya kila siku. Kwa upande wa fani, mtafiti alionesha jinsi vipengele hivyo vinavyoweza kuakisi maisha ya wanajamii, hasa kwa kupitia matumizi ya lugha na mitindo ya waandishi. Utafiti wa Mahenge ulikuwa ni msaada mkubwa katika utafiti wetu wa maandishi ya vyoo kwa sababu chooni nako ni sehemu za umma. Hata hivyo, mandhari ya chooni na daladala ni tofauti. Hivyo, ilitazamiwa kuwa hata matumizi ya lugha ni tofauti, ingawa yangeweza kufanana kwa sehemu fulani. Utafiti huu ulifanywa ili kubainisha dhamira za maandishi ya vyooni na nafasi yake kwa jamii.

Fischer (2009) alifanya utafiti wake kuhusu mawasiliano yanavyoweza kufanyika kwa kutumia maandishi ya vyooni. Utafiti wake aliufanya katika vyoo vya wanawake. Fischer aligundua mijadala mbalimbali inayohusu masuala ya kidini, mapenzi na uhusiano (urafiki). Katika mawasiliano hayo, lugha inayotumika ni lugha ya kishairi, lugha ya nathari, maswali, majibu na mara chache, kuna mafumbo. Alihitimisha kwa kupata grafiti zenye maandishi yenye adabu, na yenye kutoa mwelekeo wa uhuru wa maamuzi kwa wasiojiamini. Alifanya utafiti wake kwa kuegemea kwenye kipengele cha mawasiliano na uharabu. Utafiti huo haukuwa wa kifasihi, lakini unatoa mwanga na mwelekeo mzuri katika makala haya.

\subsection{Dhamira za Maandishi ya Kuta za Vyooni}

Msokile (1992) anasema kuwa dhamira ni mawazo yanayoelezwa katika kazi ya sanaa. Naye Wamitila (2003) alidai kwamba dhamira ni istilahi ambayo hutumiwa kuelezea wazo kuu katika hadithi au riwaya, tamthiliya, hadithi fupi, shairi, na kadhalika. Wazo hilo huhusiana na 
lengo la mwandishi wa kazi hiyo. Pia huweza kutumiwa kueleza lengo kuu la utunzi. Katika Fasihi kuna vipengele mbalimbali ambavyo ndivyo vijenzi vya maana ya utungo na hufanya kazi ya kuumba dhamira. Kwa hakika, suala la dhamira kama kiini cha tungo za kisanaa ndilo linalosawiriwa ndani ya kazi ya Fasihi. Katika utafiti wetu tumewasilisha na kuchambua dhamira mbalimbali zitokanazo na maandishi ya chooni kama ifutavyo:

\subsection{Kuhusu Dini}

Waumini wa dini za Kikristo na Kiislamu, wote kwa pamoja, wameonesha imani zao kwa kuasa na kuelimisha watu katika maandishi ya chooni. Kwa dini zote mbili, hofu ya maisha na kifo imejitokeza. Waandishi wa maandishi hayo wanasema, "Swali kabla hujaswaliwa kifo mbele yetu." Pia mwingine anasema, "Moto uko tayari, heri uokoke leo" kabla hujaifikia hukumu ya maneno yako! Mafundisho ya dini zote yanaonesha kuwa duniani tunapita tu, hivyo ni vema watu wakafanya yale yanayompendeza Mungu. Kwa kufanya matendo mema utakuwa na uhakika kuwa baada ya maisha ya hapa duniani utakuwa wapi. Mwandishi mwingine anauliza, "Yesu anakuja; utakuwa wapi baada ya kumaliza kipindi chako cha kuishi hapa duniani? Mbinguni au Jehanamu?" Pia waumini wa dini ya Kikristo wanasisitiza juu ya kumtegemea Yesu. Wanasema, "Hakika Yesu ni jibu"; "Mwombe Yesu akuongoze katika matendo"; "Yesu ni mwokozi wa ulimwengu. Mpe Yesu maisha yako nawe utakuwa salama."

Pia, kuna uhimizaji wa kumwabudu Mungu kwa kusema kuwa "siku za mwisho zinakaribia," "ni nyakati za rehema." Waandishi mbalimbali wanasema, "Okokeni, ni nyakati za rehema"; "Heri tu ungeokoka sasa"; "Heri ungeokoka leo ili makosa yasitiriwe mbele za Mungu, kwani saa ya wakovu ni sasa." Waandishi wanahimiza kila jambo kufanyika kwa wakati wake. Hivyo, wasomaji kama watafuata maelezo hayo yatawasaidia, kwani kama waandishi wa maandishi ya vyooni wanasema kuwa kila jambo na wakati wake, basi hatuna budi tumwombe sana Mungu.

\subsection{Kuhusu Maradhi}

Katika jamii mbalimbali watu wamekuwa wakiogopa sana kupata ugonjwa wa UKIMWI, ingawa wengi wanajua namna ugonjwa huo unavyoambukizwa bado hawachukui tahadhari, wanaendelea kufanya mapenzi yasiyo salama. Waathirika wengi wa ugonjwa huu wamekuwa wakiona aibu kujitangaza hadharani, kwani wapo watu wanaoona muathirika wa ugonjwa huu ni mzinzi, hivyo inaonekana ni ugonjwa wa aibu. Katika utafiti wetu tuliona watu wakitoa hisia zao kwa kusema kuwa wameathirika. Mwandishi mmoja anasema, "UKIMWI unanimaliza." Mwandishi huyu anatoa hisia zake sehemu ambayo mtu hatamjua, yawezekana anatarajia mchango wa mawazo kutoka kwa watu ili ajue nini cha kufanya. Ametumia lugha ya kusononesha. Mwandishi mwingine naye ameandika, "Nina ngoma." Lugha aliyoitumia mwandishi wa mwanzo na wa pili ni tofauti, ingawa lengo ni moja kuelezea hisia zao kama wana ugonjwa wa UKIMWI. Mwandishi wa pili anatumia lugha ya mtaani ambayo si lugha rasmi, tofauti na mwandishi wa mwanzo. Kimsingi lugha zote zinafikisha ujumbe wa aina moja. 
Mwandishi mwingine anawaasa vijana kwa kusema, "Vijana... mapenzi siyo ngono - AIDS KILL." Mwandishi huyu anaona kuwa vijana wengi wa jinsia tofauti wakiwa katika mahusiano ya urafiki wanadhani kuwa ni lazima wajihusishe na tendo la ngono. Ndiyo maana anawaasa kuwa UKIMWI unaua.

Katika kuendelea kuzungumzia ugonjwa wa UKIMWI mwandishi mwingine anaandika "Msiopiga puli mtapata ngoma." Kupiga puli ni neno lisilo rasmi lenye maana ya kupiga punyeto/kujichua. Hivyo mwandishi huyo ameandika inawezekana akawa anamaanisha kwamba watu wakipiga punyeto hawatakuwa na hamu ya kufanya ngono kwa kuwa punyeto inawaridhisha na hivyo kuepuka UKIMWI.

UKIMWI kama adhabu ni dhamira nyingine inayoibuka katika maandishi ya chooni, dai ambalo tunadhani si la kweli. Hii inajitokeza katika majibizano ambayo tumeyapatia herufi a, b, na c. Maandishi haya yameandikwa na watu tofauti na nyakati tofauti kwa mtindo wa majibizano:
(a) Kuma tamu
(b) UKIMWI, je?
(c) Adhabu yake. 
Dhamira hii inaonesha jinsi baadhi ya watu walivyo na mtazamo ambao si sahihi kuhusu ugonjwa wa UKIMWI, kwamba ni sawa na adhabu. Watu wanapaswa kutambua kwamba ugonjwa wa UKIMWI unaweza kuupata kwa njia mbalimbali, na aliyepata ugonjwa huu siyo kwamba ametenda kosa linalostahili kuadhibiwa kwa njia hiyo.

\subsection{Kuhusu Elimu}

Elimu ni kitu muhimu katika maisha ya mwanadamu. Katika jamii, elimu hutolewa kwa namna mbalimbali. Kuna elimu inayotolewa katika mfumo maalumu na ile isiyotolewa katika mfumo maalumu, lakini zote lengo lake ni kuelimisha ili mtu aweze kupambana na mazingira yanayomzunguka. Katika utafiti wetu tumepata dhamira zinazozungumzia elimu kama ifuatavyo:

"Urithi wa mtoto ni elimu, siyo majumba." Mwandishi huyu anasisitiza kwa wazazi au walezi kuwa kitu cha muhimu katika maisha ni kumuelimisha mtoto, na si kumjengea mazingira ambayo yatamfanya ategemee mali za wazazi bila yeye kujishughulisha. Katika jamii tumeona watu wengi ambao wanategemea mali za urithi na kisha wakashindwa kuzisimamia na, hatimaye, mali hizo hupotea. Mwandishi mwingine ameandika, "Elimu siyo kupanda vidato." Katika msemo huo dhamira hii ya elimu inajitokeza pale ambapo mwandishi anasisitiza kuwa kuelimika si kusoma sana, kwani wapo waliosoma sana na wakafanya mambo ambayo hayaisaidii jamii.

Maandishi mengine yanasema, "Siku Polisi wakielimika, dola lipo matatani." Polisi ni chombo cha dola ambacho husimamia na kulinda haki na mali za raia na nchi. Mara nyingi polisi hutumia nguvu katika kupambana na raia endapo kunatokea mtafaruku baina ya raia dhidi ya serikali. Wakati mwingine raia huwa wanatetea maslahi ya umma ambapo na polisi mwenyewe ni mmoja kati ya watu wanaotetewa, lakini kwa kuwa polisi ni mtu wa kutekeleza amri, akipewa amri ya kutuliza ghasia, hutekeleza amri hiyo. Kwa mtazamo wa mwandishi anaona kwamba polisi hawajaelimika kwa kuwa hutekeleza amri bila kuhoji. Lakini ukweli ni kwamba shughuli za kijeshi ni za kiimla/kidikteta, na wala si za kidemokrasia. Si kila muktadha katika maisha huhitaji demokrasia.

\subsection{Maadili na Utamaduni}

TUKI (2006) wanaeleza kuwa maadili ni mwenendo mwema. Katika utafiti wetu tumeona jinsi maadili mema yanavyozungumziwa katika maandishi hayo. Kwa mfano, kuna mwandishi anasema, "Acha kutembea na wake za watu." Mwandishi huyu anatoa onyo kwa kuhimiza kuacha tabia ya kutembea (kufanya mapenzi) na wake za watu. Katika Kiswahili, kuna msemo usemao kuwa mke wa mtu ni sumu, msemo ambao unamaanisha kwamba ukitaka kufa fanya mapenzi na mke wa mtu, ndiyo maana mke anafananishwa na sumu.

\subsection{Matabaka}

Matabaka yapo katika jamii zote duniani, ambapo kundi la watu wenye hali moja linatokana na jamii yenye mfumo wa kiuchumi ambao hugawanya watu kufuatana na uwezo wao wa kumiliki na kutawala njia za kuzalisha mali. Kundi hilo lenye nguvu ndilo ambalo mara nyingi hunyonya kundi la wasionacho katika sekta mbalimbali (Shivji, 2006, 2009). Hali hii inafanya kuwe na misemo mingi katika jamii ambayo nayo inajitokeza katika maandishi ya chooni. Kwa mfano, "Sheria duniani, haki mbinguni." Msemo huu unaibua dhamira ya matabaka katika jamii ambapo wenye nacho sheria huwa upande wao; hata wakitenda kosa hawawezi kupata hukumu itakayowaumiza, kwani wakati mwingine sheria hupindishwa na wao kuonekana hawana kosa. 
Lakini asiyenacho hali huwa tofauti, hata kama hana kosa huweza kupewa kosa. Mwisho anaishia kusema kuwa tumwachie Mungu ambapo ndipo haki itapatikana baada ya sheria kutokuwa rafiki yake.

"Dunia ya Mungu, mali za Mzungu" ni msemo ambao unaibua dhamira ya matabaka. Unaonesha kwamba mzungu ana nguvu zaidi ya watu wengine duniani. Msemo huu yawezekana umeibuka baada ya kuona jinsi mataifa ya Ulaya toka karne nyingi yalivyoweza kutawala sehemu kubwa ya dunia, na kuweza kupora mali nyingi. Katika bara la Afrika walichukua mali ghafi mbalimbali. Hadi leo, mataifa ya Ulaya ndiyo yenye nguvu na yanaendeleza ukoloni mamboleo (Adedeji, 1993; Mkandawile, 2001; Shivji, 2009).

\subsection{Mapenzi}

Kazi nyingi za Fasihi zinagusia dhamira ya mapenzi, ambayo yaweza kuwa mapenzi ya mtu na mpenzi mke, au hata mtu na ndugu au jamaa zake, au hata mapenzi ya mtu kwa nchi yake. Katika utafiti wetu, maandishi tuliyokutana nayo yanaeleza mapenzi kama ifuatavyo: Mwandishi mmoja anaeleza ugumu wa kupima upendo kwa kusema, "Upendo haupimwi kwa nguvu za macho". Dhamira hii inatahadharisha kuwa makini katika kutambua upendo, kwani yupo anayeweza kusema anakupenda kwa kukuonesha mapenzi, nawe ukaliona hilo, lakini ni vigumu kutambua ndani ya moyo wake mtu huyo anawaza nini kuhusu wewe. Mwandishi mwingine anauliza swali na mtu mwingine akalitolea jibu, anasema: "Kwanini demu wako anampenda sana rafiki yako?" na jibu linalotolewa ni "Sababu (rafiki yangu) anahonga sana." Swali na jibu hilo linaonesha dhamira nyingine ya mapenzi inayojitokeza hapa kuwa ni kukosekana kwa uaminifu baina ya wapenzi na kunatokana na tamaa ya pesa. Mapenzi siku hizi yametawaliwa na pesa, ambapo mwenye pesa ndiye anayepata mpenzi hata kwa kuchukua mke au mume wa mtu. Dhana ya mapenzi haiishii hapo, mwandishi mwingine ameandika, "Chuki ni uchafu wa nafsi," usemi ambao unaonesha kukosekana kwa mapenzi baina ya mtu na mtu. Mtu mwenye chuki daima hana mapenzi kwa yule anayemchukia. Na katika dunia ya leo chuki imetawala kwa baina ya mtu na mtu, taifa kwa taifa, dini kwa dini, na kadhalika. Hali hii inasababisha watu hata kuuana. Hivyo, ni dhahiri kuwa mtu asiye na mapenzi nafsi yake si safi.

\subsection{Umuhimu wa Lugha Yako}

"Mkataa kwao ni mtumwa," ni msemo wa Kiswahili unaohimiza watu kutosahau walikotoka. Kila lugha hapa ulimwenguni ni bora, kwani hakuna lugha ambayo ni bora zaidi ya nyingine. Hapa kwetu Tanzania kuna baadhi ya watu ambao wanaona kuzungumza Kiingereza humfanya mtu kuonekana bora zaidi ya mwingine, kitu ambacho si kweli. Katika kulionesha hili msemo ufuatao unadhihirisha hili, mwandishi anasema, "Kutojua lugha ngeni siyo ujinga." Hapa mwandishi anasisitiza kuwa matumizi ya lugha yako ni bora zaidi ya lugha nyingine.

Kutokana na dhamira hizo tunaona kwamba maandishi ya vyooni yana umuhimu katika jamii kutokana na uwezo wake wa kuelimisha juu ya mambo mbalimbali katika jamii kama vile UKIMWI, umuhimu wa kumuamini Mungu, Umuhimu wa mapenzi katika jamii, na tahadhari ya kuwa makini katika kupima upendo, umuhimu wa elimu katika jamii, kuanzia kwa watoto hadi watu wazima na kuthamini lugha yako.

\section{Nafasi ya Dhamira ya Maandishi Vyooni katika Jamii}




\subsection{Ubunifu na Falsafa ya Dini}

Dini ni miongoni mwa vipengele muhimu sana katika maisha ya mwanadamu. Kutokana na dini ya jamii fulani, falsafa ya jamii huundwa na hata maadili pia hufafanuliwa. Aidha, kuna mawazo mbalimbali kuhusu maana na umuhimu wa suala la dini katika jamii. Kwa mfano, waumini wa dini ya Kiislamu wanaamini kuwa dini ni mfumo wa maisha na kutokana na dhana hii dini inatarajiwa kuhusisha vipengele vyote vya maisha. Mojawapo ya fasili ya maana ya dini ni ile inayosema kuwa dini ni mfumo/utaratibu fulani wa maisha unaofuatwa na jamii fulani ya wanadamu (www.uislamu.org/utangulizi.htm).

Dhana hizi mbili, yaani dini kama mfumo wa maisha yanayofuatwa na jamii fulani na kuwa katika dini kitu muhimu ni maisha baada ya kifo, pia yanawiana na namna Wabantu wanavyoitazama dini. Mwanafalsafa Temples (1952) anathibitisha hili kwa kusema kuwa Wabantu wanaamini uwepo wa Mungu, na wanaamini kwamba kuna maisha baada ya kifo.

Mafunzo haya kuwa Mungu yupo, kuna maisha baada ya kifo na kwamba sala ndiyo nguzo muhimu kwa mwanadamu tunayaona pia katika data zetu. Katika utafiti wetu kuna misemo kadhaa ambayo inahusiana na suala la dini na kifo. Misemo hiyo ni kama vile "swali kabla hujaswaliwa kifo mbele yetu," "swala siyo kinga ya kifo" na "kifo na swala ni vitu viwili tofauti." Mafunzo yaliyomo kwenye misemo hii tunaweza kuyahusisha na mtazamo wa jamii kuhusu maana ya dini, umuhimu wa maisha baada ya kifo na uwepo wa Mungu. Miongoni mwa mafunzo hayo tunayoyapata kupitia maandishi ya chooni ambayo katika kazi hii yamejadiliwa kama sehemu ya kazi za kibunifu ni maisha baada ya kifo, kufanya ibada, uwezekano wa kumuombea dua mtu aliyekufa na kifo ni lazima.

Kutokana na umuhimu wa dini katika maisha, wanajamii hawaoni uzito kukariri na kutamka maneno yanayoendana na dini hata sehemu za chooni. Kwa maana hiyo basi, wigo wa kueneza elimu ya dini ni mpana kiasi kwamba wanadamu wanaambatana nayo katika maeneo yote na sehemu zote za kazi, nyumbani na hata wakienda chooni.

\subsection{Ubunifu na Suala la Maarifa}

Katika kipengele hiki tumejadili kuhusu umuhimu wa elimu katika jamii na mtazamo wa maarifa kwa jumla. Mjadala kuhusu elimu katika jamii na jinsi inavyohusiana na masuala ya kiubunifu kama yalivyojitokeza katika utafiti wetu ni muhimu kwa sababu maarifa hayo yanasaidia, siyo tu katika kuelezea mtazamo wa jamii husika kuhusu maarifa, lakini pia jambo hili linadokeza mtazamo jamii kuhusu ukweli wa maisha.

Akiandika kuhusu uhusiano baina ya maarifa, ukweli wa maisha na matendo au uzoefu, Madumulla (1993, uk.142) anasema:

Katika nadharia ya falsafa ya ufahamu sheria ya msingi ya ukweli iko katika matendo. Matendo (au uzoefu) ni msingi na lengo la ufahamu (maarifa), na ni kigezo cha ukweli.

Kutokana na dondoo hilo hapo juu, falsafa ya ukweli, ufahamu na uzoefu wa mwanadamu yanafungamana na kwamba, yote kwa pamoja, yanatusaidia pia kuhusianisha na nafasi ya sanaa katika jamii. Katika utafiti wetu kuna mfano mmoja unaohusu jambo hili. Mfano huo tunaupata katika msemo usemao kuwa, "elimu si kupanda vidato." Kutokana na msemo huu, tunapata 
mawazo kuwa jamii na kundi la watu waliofanyiwa utafiti linaamini kuwa maana halisi ya elimu si kuwa na vyeti vingi, bali elimu sahihi itapimwa kutokana na matendo ya mhusika. Kwa mantiki hiyo basi, elimu au maarifa sahihi yanategemea kiwango cha utendaji kazi alichofikia mtu. Baadhi ya watu wanaweza kuwa ama hawajasoma elimu ya namna yoyote au wamesoma, lakini wana kiwango kidogo cha elimu, ijapokuwa watu hao ni watendaji wazuri na watekelezaji bora wa majukumu yao kuliko hata wale ambao wanahesabika kuwa ni wasomi na wataalamu wa fani mbalimbali.

Kwa hivyo basi, kutokana na matokeo ya utafiti huu ni dhahiri kuwa matendo na uzoefu wa mwanadamu ni lengo na kigezo cha maarifa. Ubora na udhaifu wa elimu aliyo nayo mtu unatarajiwa kupimwa kutegemea kiwango cha ufanisi katika suala la kuwajibika.

\subsection{Ubunifu, Maadili na Utamaduni}

Utamaduni ni jumla ya mambo yote yanayobuniwa na jamii ili kukidhi utashi na maendeleo yake. Kwa maneno mengine, utamaduni ni mwenendo wa maisha ya jamii, mtazamo wao wa mambo, na taratibu zao za kuendesha maisha zinazowatofautisha wao na jamii nyingine. Katika utamaduni kuna vitu kama vile lugha, mila na desturi hata maadili. Kimsingi, dhana za ubunifu, maadili na utamaduni zinahusiana kwa kiwango kikubwa. Katika utafiti wetu, kuna mambo kadhaa ambayo yanaashiria kuwa sanaa ya kuandika katika vyoo ni sehemu ya ubunifu wa wanajamii katika jamii ya Watanzania na jamii nyinginezo. Aidha, katika ubunifu huu suala la kimaadili linalojitokeza kwa sura tofauti tofauti. Sura ya kwanza ni mchakato mzima wa kuandika chooni, na sura ya pili ni matumizi ya lugha safihi.

Katika utafiti wetu, tulibaini kuwa idadi kubwa ya maandishi katika maeneo ya chooni ilitumia lugha safihi. Katika jamii ya wanadamu si kawaida kuona watu wanatumia lugha ya namna hiyo. Lakini katika maandishi tuliyochunguza, jambo hili lilikuwa la kawaida sana. Miongoni mwa misemo hiyo inayodokeza na kutumia lugha ya matusi ni pamoja na "okokeni kuma nyoko nyie," "kufirwa ni bomba," "napenda kufira," na kadhalika. Kutokana na hali hii tunaweza kuhitimisha kwa kutoa dondoo chache.

Kwanza, kwa kuwa si kawaida kutumia lugha safihi, wanajamii hutumia fursa yao pindi wawapo katika maeneo ya chooni kueleza hisia zao. Kwa hiyo, katika Fasihi, kuna fursa ambayo inampa mwanadanu uhuru wa kunena na kutenda zaidi ya yale ambayo hayatarajiwi kutokea. Matakwa na utashi wa mwanadamu hujidhihirisha zaidi katika sanaa, hususani Fasihi, kuliko katika tasnia nyingine yoyote. Hivyo, maandishi haya yanaonesha asili ya 'unyama' au uhayawani iliyojificha kwa mwanadamu. Mwanadamu huyu afanyapo uhayawahi huo ndipo roho/nafsi yake huridhika.

Pili, kutokana na matokeo ya utafiti huu, sehemu mojawapo na mahali pa kuweza kumfahamu mwanadamu kwa usahihi ni faraghani. Hii ni sehemu ambayo mwanadamu yuko peke yake, haathiriwi na mihemko wala matakwa ya jamii. Hivyo, huwa ameitoroka mihemko na kupata muda wa 'kiasi' wa kupambana na mihemko hiyo kwa kificho, akichelea kuwa akijitokeza katika uwazi atadhibitiwa. Aidha, ana kuwa hayuko katika upweke, kwa sababu anajua anapoandika haandikii ombwe, bali jamii. Na anajua kama hatajibiwa, wamesikia, 'wataipata.' 


\subsection{Hitimisho}

Katika makala haya tumewasilisha na kujadili matokeo ya utafiti kuhusu dhamira ya maandishi ya vyooni na nafasi yake katika jamii. Kutokana na matokeo tuliyojadili, imebainika kuwa ubunifu unaojitokeza katika maandishi maeneo ya vyooni umefumbata mbinu maridhawa za kisanaa. Matokeo ya utafiti huu yameonesha kuwa katika maandishi tuliyoyatafiti kuna dhamira mbalimbali na ambazo zinahusiana na mambo yaliyopo katika jamii. Dhamira zilizojadiliwa zilikuwa ni maradhi, maarifa, maadili, matabaka, umuhimu wa lugha yako, mapenzi na suala la dini. Pia nafasi ya dhamira hizo katika jamii imejadiliwa.

\section{Marejeo}

Adedeji, A. (1993). Africa within the World: Beyond Dispossession and Dependence. London: Zed books.

Babbie, E (1999). The Basics of Social Research. Belmont: Wadsworth Publishing Company.

Crewswell, J. W. (2009). Research Design: Qualitative, Quantitative, and Mixed Methods Approaches. Los Angeles: SAGE Publications.

Enkvist, N. E. (1973). Linguistic stylistics, Mouton: The Hague \& Paris.

Fischer, K. (2009). Latrine Literature. http://www.dw.com/en/german-student-gets-degree-inlatrine-literature/a-4540624, Jumanne, 01-03-2011.

Isaac and Michael, (1981). Handbook of research and evolution: A collection of principles, methods and strategies useful in the planning, design and behavioural science, San. Diago: Edips.

Kothari, C. R. (1992). Research Methodology: Methods and Techniques. New Delhi: Wiley Eastern Limited.

Kothari, C. R. (2008). Research Methodology: Methods and Techniques. New Delhi: New Age International Publishing Ltd.

Madumulla, J. S. (1993). Mwandishi wa Riwaya ya Kiswahili na Suala la Ukweli wa Maisha: Fasihi, Uandishi na Uchapishaji, Dar es Salaam: DUP.

Mahenge, E. (2009). "Uchambuzi wa Fani na Maudhui katika Semi zilizokwenye tiketi za Daladala: Dar es Salaam” Kioo cha Lugha, Juzии 7, Dar es salaam, TATAKI.

Miall, D. S., \& Kuiken, D. (1994). Poetics, University of Alberta.

Mkandawire, T (1995). "Beyond Crisis: Towards Democratic Development State in Africa", Dakar: CODESRIA Eighth General Assembly.

Msokile, M. (1992). Misingi ya Hadithi Fupi, Dar es Salaam, DUP.

Mutembei, A. K. (2005). Beyond the Similaritude of AIDS to the Miscellany of its culture A Comparison of Figurative Language on AIDS Billboards across Africa" (Haijachapishwa).

Shivji, I. G. (2006). Let the People Speak: Tanzania down the road to neo-liberalism, Dakar: CODESRIA.

Shivji, I. G. (2009). Accumulation in an African Periphery: A Theoretical Framework. Dar es Salaam: Mkuki na Nyota.

Tempels, P. (1954). Bantu Philosophy, Paris. Presence Africaine.

TUKI. (2006). Kamusi ya Kiswahili Sanifu, Nairobi. O.U.P.

Uislamu.org (2011). Utangulizi wa Dini na Uislamu, http://www.uislamu.org/utangulizi.htm Jumatano, 09-06-2011.

Wamitila, K. W. (2003). Kamusi ya Fasihi Istilahi na Nadharia, FPL, Nairobi. 